\title{
Embryonic-maternal cross-talk via exosomes: potential implications
}

This article was published in the following Dove Press journal:

Stem Cells and Cloning: Advances and Applications

7 July 2015

Number of times this article has been viewed

\author{
Islam M Saadeldin' \\ Hyun Ju Oh \\ Byeong Chun Lee 2,3 \\ 'Department of Physiology, \\ Faculty of Veterinary Medicine, \\ Zagazig University, Zagazig, Egypt; \\ ${ }^{2}$ Department of Theriogenology and \\ Biotechnology, College of Veterinary \\ Medicine and the Research Institute \\ for Veterinary Science, Seoul National \\ University, Seoul, Republic of Korea; \\ ${ }^{3}$ Institute of Green Bio Science and \\ Technology, Seoul National University, \\ Pyeong Chang, Kangwon do, Republic \\ of Korea
}

Correspondence: Byeong Chun Lee Department of Theriogenology and Biotechnology, College of Veterinary Medicine and the Research Institute for Veterinary Science, Seoul National University, I Gwanak-ro, Gwanak-gu, Seoul, I5I-742, Republic of Korea

Tel +8228801269

Fax +82 $2873 \quad 1269$

Email bclee@snu.ac.kr

\begin{abstract}
A myriad of locally produced factors into the microenvironment of the reproductive tract is regulated, not one-way but rather, through embryonic-maternal cross-talk. In this minireview, we focused on the exosomes, which are cell-derived vesicles of 30-100 nm in diameter, as a communicating language facilitating this dialog. These nanovesicles are secreted from preimplantation embryos, oviduct epithelium, and endometrium as well as from the placenta, and contain proteins, messenger RNA (mRNA), microRNA, and DNA cargoes, and have pleiotropic effects on both embryonic and maternal environments. A better understanding of the molecular mechanisms mediating this cross-talk will lead to the development of new regulating agents, with novel diagnostic, biological, and therapeutic potential for either supporting or hindering the normal reproductive functions.
\end{abstract}

Keywords: embryo, endometrium, placenta, mRNA, miRNA

\section{Exosomes}

An ever growing number of studies worldwide have helped to substantiate the essential functions of the cell-secreted, membrane-derived vesicles, particularly exosomes, and provided new dimensions for the concept of intercellular signaling. Exosomes are nanosized vesicles (30-100 $\mathrm{nm}$ in diameter) (Figure 1A and B) that contain, not only proteins, but also, messenger RNAs (mRNAs), microRNAs (miRNAs), and double-strand or genomic DNA. ${ }^{1-6}$ The molecular cargoes carried by exosomes affect cellular activity via ready-made proteins and miRNA or by translation of transferred mRNAs (Figure 1C). The term "exosomal shuttle RNA (esRNA)" was proposed for those transferred RNAs. ${ }^{3}$

Exosomes, as cell membrane-derived nanovesicles, are specifically equipped to mediate intercellular communication, via the transfer of genetic information to recipient cells. As a result, exosomes play a fundamental biological role in the regulation of normal physiological as well as aberrant pathological processes, via altered gene regulatory networks and via epigenetic programming. ${ }^{2}$ For example, exosome-mediated genetic transfer can regulate the maintenance of stem cell plasticity and induce beneficial cell phenotype modulation. ${ }^{7}$ Alternatively, such vesicles play a role in tumor pathogenesis $^{8}$ and the spread of neurodegenerative diseases, via transfer of specific miRNAs and pathogenic proteins. ${ }^{9}$ In addition, these cell membrane-derived vesicles are involved in cell adhesion and signal transfer, and provide an important means of cell communication. ${ }^{10}$ Evidence of secretion of exosomes has been reported in most cell types, including embryonic stem cells and in vitro-produced embryos. ${ }^{11-16}$ 


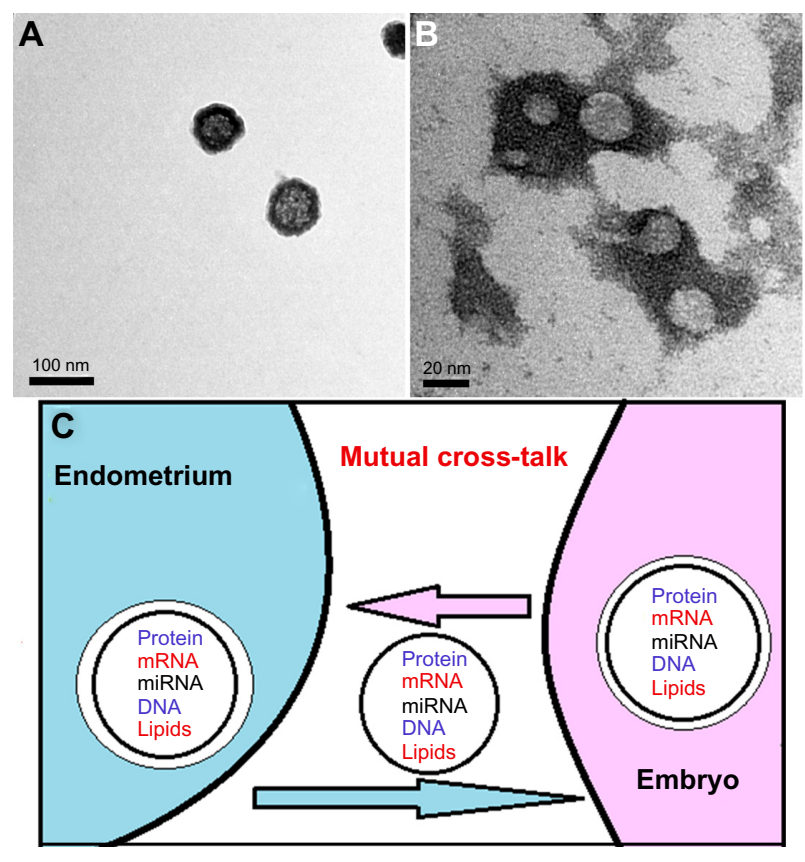

Figure I Embryo-derived exosomes as seen by transmission electron microscope. Notes: TEM images show the presence of particles, ranging from less than $100 \mathrm{~nm}$ (A) to $30 \mathrm{~nm}(\mathbf{B})$, in an embryo-conditioned medium pellet isolated by differential centrifugation, after negative staining with uranyl acetate (more detailed methods for exosomes isolation are described in our previous report). ${ }^{14} \mathrm{~A}$ sketch showing the paracrine intercellular communication between embryos and endometrium, through the exosomes $(\mathbf{C})$.

Abbreviations: mRNA, messenger RNA; miRNA, microRNA; TEM, transmission electron microscope.

Because of the ever increasing discoveries in the field of extracellular vesicles, Vesiclepedia (http://microvesicles. org) has been established as a compendium database for extracellular vesicles and exosomal components, including proteins and RNAs. ${ }^{17}$

The early stages for pregnancy require preparatory cross-talk and signals. Of interest are the exosomes from both embryonic and maternal sides, by which the maternal recognition of pregnancy will be achieved successfully. ${ }^{18}$ In the coming section, we give a brief description about the probable and/or proposed involvement of exosomes in mediating the embryonic-maternal cross-talk.

\section{Embryo-derived exosomes}

In study of the physical properties, including size and concentration, of in vitro fertilized (IVF) embryos-derived exosomes, it was revealed that their numbers increase with developmental stage and further, that their size correlates with embryo quality and may predict recovery from apparent growth-arrested embryos. ${ }^{13}$

Moreover, in previous work, we showed the uptake of embryo-derived exosomes by cultured embryos. These exosomes were shown to act as a mediator, carrying early reprogramming mRNAs, such as Oct4, Sox2, cMyc, and Klf4, which improved the development of the cocultured embryos in group culture system. ${ }^{14}$ We suggest that the continuous transfer of mRNA cargoes among cultured embryos via exosomes is advantageous over the acute transfer of mRNA by the conditioned medium - this confirms the concept of the "dynamic microenvironment" or "niche" among the cultured embryos. It was suggested that the stability of foreign mRNAs in cells is often tightly and intricately regulated with low transcriptional rates. ${ }^{19}$ The foreign transferred mRNAs are rapidly turned over with half-lives of $20-40$ minutes..$^{20}$ In our preliminary work, we found expression of sex determining mRNAs, Xist and Sry, in the conditioned medium of in vitro-derived embryos cultured individually (unpublished data), which could be used for sexing of in vitro-produced embryos.

In equines, a previous in vitro study suggested that exosomes can be secreted by day 8 embryos, which can modulate the functions of the oviduct epithelium through transfer of early pregnancy factor (HSP10) and miRNAs. ${ }^{21}$

miRNAs, small ( $\sim 22$ nucleotides) noncoding RNAs that regulate gene expression, have been implicated in a wide array of biologic processes, including early embryo development and stem cell differentiation. ${ }^{22-25}$ Human blastocysts express miRNAs, which may be important to their survival. Differential miRNA expression between euploid and aneuploid embryos may be an early indicator of their prognosis or a mechanism behind their eventual fate. ${ }^{23}$ Interestingly, miRNAs have also been found to exist in embryo culture medium, in both human and domestic animals. ${ }^{15,16}$

The expression of miRNA in culture medium has been correlated to fertilization method, chromosomal status, and pregnancy outcome, which makes miRNA a potential biomarker for predicting IVF success. ${ }^{16}$ Some miRNAs, including miRNA-191, have been shown to be putative noninvasive candidates for predicting aneuploidy, while miR-191, miR-372, and miR-645 were found to be more highly concentrated in media from failed IVF/ non-intracytoplasmic sperm injection (ICSI) cycles. ${ }^{16}$ Additionally, miRNAs were found to be more highly concentrated in ICSI and day 5 media samples when compared to regularly inseminated and day 4 samples, respectively. ${ }^{16}$ Furthermore, it was shown that differential miRNA gene expression analysis could be used to early discriminate between embryos that developed to the blastocyst stage and those that failed to develop from the morula to blastocyst stage, deemed degenerate embryos. miR-25, miR-302c, miR-196a2, and miR-181a expression was found to be higher 
in degenerate embryos compared to blastocyst embryos. ${ }^{15}$ Therefore, expression of miRNAs in in vitro culture media could allow for the development of biological markers for selection of better quality embryos and for subsequent successful pregnancy.

Furthermore, embryonic and fetal genomic DNA was also found to circulate in the maternal blood. ${ }^{26,27}$ This raises many questions about the involvement of exosomes/ microvesicles in transportation of embryonic and fetal genomic DNA ${ }^{4,28,29}$ and may mean that these exosomes can act as potent markers, specifically, for the Y-chromosome specific DNA; for embryo/fetal sexing as early as 6 weeks of gestation; ${ }^{26,30-32}$ or as a noninvasive prenatal genetic diagnostic method to detect aneuploidy in the embryo and fetus. ${ }^{6,33-35}$

\section{Maternal-derived exosomes}

On the other hand, exosomes can be secreted from maternal side; bioinformatic analysis of endometrium-derived exosome miRNAs revealed that these miRNAs have potential targets in biological pathways highly relevant for embryo implantation. ${ }^{36}$ Western blot analysis demonstrated that in vivo-derived exosomes contain HSP70 and OVGP, which have roles in fertilization and early pregnancy; however, in vitro-derived exosomes contain only HSPA8 and MYH9. ${ }^{37}$ They are also secreted from the endometrium and have been found to contain mRNAs for ovine enJSRV-envelope, HSP70, interleukins, and IFN-regulatory factors, thereby stimulating trophectoderm cells to proliferate and secrete IFNT for successful pregnancy recognition in sheep. These results would explain that free and/or exosomal enJSRV acts on the trophectoderm, via TLRs, to induce the secretion of IFNT. ${ }^{38}$

\section{Placenta-derived exosomes}

A previous study unraveled the important role of placentaderived exosomes; the syncytiotrophoblast constitutively and throughout the pregnancy secretes exosomes. ${ }^{39}$ More recently, exosomes have been shown to be released by trophoblasts and to carry molecules involved in placental physiology. This involves immunomodulator proteins such as fibronectin and syncytin, Wnt/ $\beta$ catenin-related molecules, galectin-3, and HLA-G; but also bioactive lipids, such as immunosuppressive PGE2; PPAR $\gamma$ ligand 15d-PGJ2; and miRNAs. ${ }^{40}$

Exosomes contain all the ingredients (ie, syncytin, phosphatidic acid, and endosomal phospholipid BMP) to promote intercellular fusion and might behave as initiators of syncythiotrophoblast formation. ${ }^{41-43}$ Moreover, the placentaderived exosomes are immunosuppressive and carry proteins and RNA molecules that, in a redundant manner, influence a number of mechanisms and promote the fetal allograft survival. $^{39,44,45}$ Specifically, isolated human placental exosomes carry NKG2D ligands, ULBPs (ULBP1-5), and MIC proteins on their surface by which they induce downregulation of the NKG2D receptor on NK, CD8(+), and gamma delta $\mathrm{T}$ cells. This, in turn, leads to reduction of their in vitro cytotoxicity without affecting the perforin-mediated lytic pathway. ${ }^{44}$ Moreover, bioactive FasL- and TRAIL-carrying exosomes, which are able to convey apoptosis, are secreted by the placenta and responsible for the immunomodulatory and protective role of the human placenta. ${ }^{45}$ Exosomes can also be used for prediction of preeclampsia: Villous cytotrophoblast exosomes have been found to be positive for HERV envelope gene proteins and to be rapidly taken up by BeWo choriocarcinoma cells in a syncytin 1- and syncytin 2-dependent manner. Syncytin 2 has also been found to be reduced in women with preeclampsia. ${ }^{43}$

Furthermore, it has been shown that trophoblast-derived exosomes containing miRNAs could mediate cross-talk between the fetoplacental unit and the mother during pregnancy. The function of placenta-derived miRNAs, particularly from the primate-specific chromosome 19 miRNA clusters, which are highly expressed in human placentas and in the serum of pregnant women, has been reviewed. ${ }^{46}$

\section{Synthetic and semisynthetic exosomes}

The application of synthetic or semisynthetic exosomes for therapeutic drug delivery is still in its infancy. Issues regarding the understanding of exosomes biogenesis, large-scale production, and in vivo interactions need to be addressed to develop successful and cost-effective exosome-based drug delivery systems. ${ }^{47}$ Few investigations have explored their potential to serve as a platform for the development of semisynthetic nanovesicles. Given their nanoscale size, potential to express targeting ligands in native conformations, and their deformable structure, exosomes offer a logical biological vesicle platform for adapting and producing semisynthetic vesicles with excellent potential for nanomedicine applications. ${ }^{48}$ No doubt, progress in synthetic biology to harnessing exosomes for therapeutic nucleic acid delivery is crucial to mimic the physicochemical properties of naturally occurring exosomes to overcome delivery challenges and to establish robust technology platforms. ${ }^{49}$ If successful, the exosomes could then transport the nucleic acid into a subsequent population of cells, including embryos, that manifest the desired effect. This piggy-back mechanism 
could be responsible for reported deep-tissue RNA or DNA effects with certain carriers, and offers novel and promising roles in interfering with or supporting the dialog between the embryos and the mother. ${ }^{50}$

\section{Conclusion}

From these novel results, we may suggest a dynamic mutual paracrine communication between both the embryonic and the maternal environments, through the exosomes, at the early stages of preimplantation embryo development. Anticipating the potential implications of the functions of the embryonicand maternal-derived exosomes is essential. Particularly, the detection and characterization of embryo-derived exosomes could be a noninvasive method for: 1) the judgment of embryo quality and implantation capability; 2) the sexing of the embryos; and 3 ) the prediction of abnormal genetic constituents of embryos, as an alternative to the invasive prenatal genetic diagnosis (PGD) method. Therefore, advancement in the physicochemical analysis of small quantities of embryo culture medium, including analysis of exosomes size (such as nanoparticle tracking analysis) ${ }^{51}$ and content of mRNA, miRNA and DNA, is crucial to substantiate these targets.

Finally, much remains to be learned concerning the communicating signals comprising the embryonic-maternal cross-talk through the exosomes, especially considering that their effects are often redundant and pleiotropic. Deepening our understanding of exosomes will be critical in understanding the physiology and the pathophysiology of the early stages of pregnancy recognition. Moreover, this study will enable the development of novel nanodiagnostics and nanotherapeutics, to maximize pregnancy rates during IVF treatment; to improve the treatment of disorders such as infertility, pregnancy loss, and preeclampsia; and possibly even to offer new methods for locally acting contraceptives.

\section{Acknowledgment}

This study was supported by IPET (grant numbers 311011 05-4-SB010 and 114059-3), MI (grant number 10048948), the Research Institute for Veterinary Science, TS Corporation, and the BK21 plus program.

\section{Disclosure}

The authors declare that there is no conflict of interest.

\section{References}

1. Théry C. Exosomes: secreted vesicles and intercellular communications. F1000 Biol Rep. 2011;3:15.

2. Lee Y, El Andaloussi S, Wood MJ. Exosomes and microvesicles: extracellular vesicles for genetic information transfer and gene therapy. Hum Mol Genet. 2012;21(R1):R125-R134.
3. Valadi H, Ekström K, Bossios A, Sjöstrand M, Lee JJ, Lötvall JO. Exosome-mediated transfer of mRNAs and microRNAs is a novel mechanism of genetic exchange between cells. Nat Cell Biol. 2007;9(6): 654-659.

4. Thakur BK, Zhang H, Becker A, et al. Double-stranded DNA in exosomes: a novel biomarker in cancer detection. Cell Res. 2014;24(6): 766-769.

5. Cai J, Han Y, Ren H, et al. Extracellular vesicle-mediated transfer of donor genomic DNA to recipient cells is a novel mechanism for genetic influence between cells. J Mol Cell Biol. 2013;5(4):227-238.

6. Kahlert C, Melo SA, Protopopov A, et al. Identification of doublestranded genomic DNA spanning all chromosomes with mutated KRAS and 53 DNA in the serum exosomes of patients with pancreatic cancer. J Biol Chem. 2014;289(7):3869-3875.

7. Aliotta JM, Lee D, Puente N, et al. Progenitor/stem cell fate determination: interactive dynamics of cell cycle and microvesicles. Stem Cells Dev. 2012;21(10):1627-1638.

8. Iero $\mathrm{M}$, Valenti R, Huber $\mathrm{V}$, et al. Tumour-released exosomes and their implications in cancer immunity. Cell Death Differ. 2008;15(1): 80-88.

9. Rajendran L, Honsho M, Zahn TR, et al. Alzheimer's disease betaamyloid peptides are released in association with exosomes. Proc Natl Acad Sci U S A. 2006;103(30):11172-11177.

10. van der Pol E, Böing AN, Harrison P, Sturk A, Nieuwland R. Classification, functions, and clinical relevance of extracellular vesicles. Pharmacol Rev. 2012;64(3):676-705.

11. Ratajczak J, Miekus K, Kucia M, et al. Embryonic stem cell-derived microvesicles reprogram hematopoietic progenitors: evidence for horizontal transfer of mRNA and protein delivery. Leukemia. 2006;20(5): $847-856$.

12. Katsman D, Stackpole EJ, Domin DR, Farber DB. Embryonic stem cell-derived microvesicles induce gene expression changes in Müller cells of the retina. PLoS One. 2012;7(11):e50417.

13. Gardiner CF, Ferriera JF, Poli M, Turner K, Child T, Sargent IL. IVF embryos release extracellular vesicles which may act as an indicator of embryo quality. J Extracell Vesicles. 2013;2:20826. Abstract.

14. Saadeldin IM, Kim SJ, Choi YB, Lee BC. Improvement of cloned embryos development by co-culturing with parthenotes: a possible role of exosomes/microvesicles for embryos paracrine communication. Cell Reprogram. 2014;16(3):223-234.

15. Kropp J, Salih SM, Khatib H. Expression of microRNAs in bovine and human pre-implantation embryo culture media. Front Genet. 2014;5:91.

16. Rosenbluth EM, Shelton DN, Wells LM, Sparks AE, Van Voorhis BJ. Human embryos secrete microRNAs into culture media - a potential biomarker for implantation. Fertil Steril. 2014;101(5):1493-1500.

17. Kalra H, Simpson RJ, Ji H, et al. Vesiclepedia: a compendium for extracellular vesicles with continuous community annotation. PLoS Biol. 2012;10(12):e1001450.

18. Tannetta D, Dragovic R, Alyahyaei Z, Southcombe J. Extracellular vesicles and reproduction-promotion of successful pregnancy. Cell Mol Immunol. 2014;11(6):548-563.

19. Rajagopalan LE, Malter JS. Turnover and translation of in vitro synthesized messenger RNAs in transfected, normal cells. J Biol Chem. 1996;271(33):19871-19876.

20. Wisdom R, Lee $\mathrm{W}$. The protein-coding region of c-myc mRNA contains a sequence that specifies rapid mRNA turnover and induction by protein synthesis inhibitors. Genes Dev. 1991;5(2):232-243.

21. Bemis LT, McCue PM, Hatzel JN, Bemis J, Ferris RA. Evidence for production of early pregnancy factor (Hsp10), microRNAs and exosomes by day 8 equine embryos. J Equine Vet Sci. 2012;32(7):398.

22. Laurent LC. MicroRNAs in embryonic stem cells and early embryonic development. J Cell Mol Med. 2008;12(6A):2181-2188.

23. Rosenbluth EM, Shelton DN, Sparks AE, Devor E, Christenson L, Van Voorhis BJ. MicroRNA expression in the human blastocyst. Fertil Sterility. 2013;99(3):855-861. e3.

24. Lingenfelter BM, Tripurani SK, Tejomurtula J, Smith GW, Yao J. Molecular cloning and expression of bovine nucleoplasmin 2 (NPM2): a maternal effect gene regulated by miR-181a. Reprod Biol Endocrinol. 2011;9:40. 
25. Tesfaye D, Worku D, Rings F, et al. Identification and expression profiling of microRNAs during bovine oocyte maturation using heterologous approach. Mol Reprod Dev. 2009;76(7):665-677.

26. Al-Yatama MK, Mustafa AS, Ali S, Abraham S, Khan Z, Khaja N. Detection of $Y$ chromosome-specific DNA in the plasma and urine of pregnant women using nested polymerase chain reaction. Prenat Diagn. 2001;21(5):399-402.

27. Lo YM, Corbetta N, Chamberlain PF, et al. Presence of fetal DNA in maternal plasma and serum. Lancet. 1997;350(9076):485-487.

28. Sarker S, Scholz-Romero K, Perez A, et al. Placenta-derived exosomes continuously increase in maternal circulation over the first trimester of pregnancy. J Transl Med. 2014;12:204.

29. Salomon C, Torres MJ, Kobayashi M, et al. A gestational profile of placental exosomes in maternal plasma and their effects on endothelial cell migration. PloS One. 2014;9(6):e98667.

30. Yasmin L, Takano J, Nagai Y, Otsuki J, Sankai T. Detection and quantification of male-specific fetal DNA in the serum of pregnant cynomolgus monkeys (Macaca fascicularis). Comp Med. 2015;65(1):70-76.

31. Kolialexi A, Tounta G, Apostolou P, et al. Early non-invasive detection of fetal Y chromosome sequences in maternal plasma using multiplex PCR. Eur J Obstet Gynecol Reprod Biol. 2012;161(1): 34-37.

32. Devaney SA, Palomaki GE, Scott JA, Bianchi DW. Noninvasive fetal sex determination using cell-free fetal DNA: a systematic review and meta-analysis. JAMA. 2011;306(6):627-636.

33. Liu FM, Wang XY, Feng X, Wang W, Ye YX, Chen H. Feasibility study of using fetal DNA in maternal plasma for non-invasive prenatal diagnosis. Acta Obstet Gynecol Scand. 2007;86(5):535-541.

34. Sekizawa A, Purwosunu Y, Matsuoka R, et al. Recent advances in noninvasive prenatal DNA diagnosis through analysis of maternal blood. J Obstet Gynaecol Res. 2007;33(6):747-764.

35. Wright CF, Burton H. The use of cell-free fetal nucleic acids in maternal blood for non-invasive prenatal diagnosis. Hum Reprod Update. 2009;15(1):139-151.

36. Ng YH, Rome S, Jalabert A, et al. Endometrial exosomes/ microvesicles in the uterine microenvironment: a new paradigm for embryo-endometrial cross talk at implantation. PLoS One. 2013;8(3): e58502.

37. Almiñana C, Corbin E, Tsikis G, et al. Characterization of bovine oviductal exosomes from in vivo and in vitro origin. Reprod Fertil Dev. 2015;27(1):147.

38. Ruiz-González I, Xu J, Wang X, Burghardt RC, Dunlap KA, Bazer FW. Exosomes, endogenous retroviruses and toll-like receptors: pregnancy recognition in ewes. Reproduction. 2015;149(3):281-291.
39. Mincheva-Nilsson L, Baranov V. The role of placental exosomes in reproduction. Am J Reprod Immunol. 2010;63(6):520-533.

40. Record M. Intercellular communication by exosomes in placenta: a possible role in cell fusion? Placenta. 2014;35(5):297-302.

41. Record M, Carayon K, Poirot M, Silvente-Poirot S. Exosomes as new vesicular lipid transporters involved in cell-cell communication and various pathophysiologies. Biochim Biophys Acta. 2014;1841(1): 108-120.

42. Laulagnier K, Motta C, Hamdi S, et al. Mast cell- and dendritic cellderived exosomes display a specific lipid composition and an unusual membrane organization. Biochem J. 2004;380(Pt 1):161-171.

43. Vargas A, Zhou S, Éthier-Chiasson M, et al. Syncytin proteins incorporated in placenta exosomes are important for cell uptake and show variation in abundance in serum exosomes from patients with preeclampsia. FASEB J. 2014;28(8):3703-3719.

44. Hedlund M, Stenqvist AC, Nagaeva O, et al. Human placenta expresses and secretes NKG2D ligands via exosomes that down-modulate the cognate receptor expression: evidence for immunosuppressive function. J Immunol. 2009;183(1):340-351.

45. Stenqvist AC, Nagaeva O, Baranov V, Mincheva-Nilsson L. Exosomes secreted by human placenta carry functional Fas ligand and TRAIL molecules and convey apoptosis in activated immune cells, suggesting exosome-mediated immune privilege of the fetus. J Immunol. 2013; 191(11):5515-5523.

46. Ouyang Y, Mouillet JF, Coyne CB, Sadovsky Y. Review: placentaspecific microRNAs in exosomes - good things come in nano-packages. Placenta. 2014;35 Suppl:S69-S73.

47. van der Meel R, Fens MH, Vader P, van Solinge WW, Eniola-Adefeso O, Schiffelers RM. Extracellular vesicles as drug delivery systems: lessons from the liposome field. J Control Release. 2014;195:72-85.

48. Hood JL, Wickline SA. A systematic approach to exosome-based translational nanomedicine. Wiley Interdiscip Rev Nanomed Nanobiotechnol. 2012;4(4):458-467.

49. Marcus ME, Leonard JN. FedExosomes: Engineering therapeutic biological nanoparticles that truly deliver. Pharmaceuticals (Basel). 2013;6(5):659-680.

50. Nguyen J, Szoka FC. Nucleic acid delivery: the missing pieces of the puzzle? Acc Chem Res. 2012;45(7):1153-1162.

51. Dragovic RA, Collett GP, Hole P, et al. Isolation of syncytiotrophoblast microvesicles and exosomes and their characterisation by multicolour flow cytometry and fluorescence Nanoparticle Tracking Analysis Methods. In press 2015.
Stem Cells and Cloning: Advances and Applications

\section{Publish your work in this journal}

Stem Cells and Cloning: Advances and Applications is an international, peer-reviewed, open access journal. Areas of interest in stem cell research include: Embryonic stem cells; Adult stem cells; Blastocysts; Cordblood stem cells; Stem cell transformation and culture; Therapeutic cloning; Umbilical cord blood and bone marrow cells; Laboratory,

\section{Dovepress}

animal and human therapeutic studies; Philosophical and ethical issues related to stem cell research. This journal is indexed on CAS. The manuscript management system is completely online and includes a quick and fair peer-review system. Visit http://www.dovepress.com/ testimonials.php to read real quotes from published authors 